\title{
OPTIMAL PORTFOLIO CONTROL WITH TRADING STRATEGIES OF FINITE VARIATION
}

\author{
BUJAR GASHI ${ }^{\star} \quad$ PARESH DATE \\ Center for the Analysis of Risk and Optimisation Modelling Applications \\ School of Information Systems, Computing and Mathematics \\ Brunel University, Uxbridge, UB8 3PH, U.K.
}

\begin{abstract}
We propose a method for portfolio selection with trading strategies constrained to having a finite variation. A linear combination of logarithms of each asset holdings values are used as a criterion, which also includes a penalty on the logarithmic rates of change of trading strategies. A simulation example shows a significant reduction in transaction cost as compared to a log-optimal portfolio.
\end{abstract}

Index Terms-Finite variation, log-optimal portfolio, no short selling, optimal control, transaction cost.

\section{INTRODUCTION}

One of the main aims in portfolio selection is optimal wealth growth. When investor is constrained to constant fractions of wealth allocated across the assets, then maximizing the log-mean of portfolio wealth is the best criterion to use for the long term investment. Such a portfolio is said to be log-optimal. These portfolios were introduced in [5] and [7] for the case of discrete-time static portfolios and more fully developed in [1]. A similar optimization problem in a market with transaction cost is given in [2]. Logoptimal portfolios in continuous-time dynamic case (which also lead to constant fractions of wealth), with constraints and transaction cost can be found in textbooks such as [6]. Having constant fractions of wealth allocated across the assets means the trading strategies will be of infinite variation. This may lead to a large transaction cost. Hence, in this paper we constrain the trading strategies to have a finite variation. This leads to a difference in wealth in comparison with the log-optimal portfolio. By minimizing such a difference, and including a penalty on the rates of change of trading strategies, we obtain explicit formulae for the number of shares to be held at each discrete time instant. We do this under the no short-selling constraint and a market with no transaction cost. Thus, this can be seen as an implicit approach to reducing the transaction cost.

The rest of the paper is organized as follows. In sec.II an introduction to the log-optimal portfolio problem and its solution is given. The finite variation constraint is introduced in sec.III, where a criterion that minimizes an upper bound of the error between such a constrained portfolio and the log-optimal one is also proposed. Formulation of the control problem to be solved, and its explicit solution are given in sec.IV. Bounds on the trading strategies are found and used in solving the problem of restricting the number of shares

\footnotetext{
^Corresponding author. Email address: bujar.gashi@brunel.ac.uk.
}

per asset in sec.V. A significant reduction in transaction cost as compared to log-optimal portfolio, is illustrated with a simulation example in sec.VI.

\section{LOG-OPTIMAL PORTFOLIO IN CONTINUOUS AND DISCRETE TIME}

We study a market consisting of a single risk-free asset and $n$ risky assets, the prices of which are given in the following standard form

$$
\begin{aligned}
& d S_{0}(t)=r S_{0}(t) d t \\
& d S_{i}(t)=S_{i}(t)\left[\mu_{i} d t+\sum_{j=1}^{n} \sigma_{i j} d W_{j}(t)\right]
\end{aligned}
$$

and $S_{i}(0)>0, i=0,1, \ldots, n$. The interest rate $r$, the drift $\mu_{i}$ and volatility $\sigma_{i j}$, are all assumed to be positive constants. The noise terms $d W_{j}(t)$ are differentials of independent standard Brownian motions. Volatility matrix $\sigma$, given by

$$
\sigma=\left\{\sigma_{i j}\right\}_{i, j=1}^{n}
$$

is assumed to be nonsingular. The trading strategy is defined as an adapted real-valued process $\left[v_{0}(t), \ldots, v_{n}(t)\right]^{\prime}$, where $v_{i}(t)$ represents the number of shares per asset. Portfolio value, i.e. the total wealth, at time $t$ is given by:

$$
y(t)=\sum_{i=0}^{n} v_{i}(t) S_{i}(t)=\sum_{i=0}^{n} y_{i}(t) .
$$

Here $y_{i}(t), i=0,1, \ldots, n$, denotes the value of the holdings per asset. These can also be expressed in terms of the fractions $\alpha_{i}(t)$ of $y(t)$ allocated to asset $i$, as follows:

$$
\begin{aligned}
y_{i}(t)=v_{i}(t) S_{i}(t) & =\alpha_{i}(t) y(t), i=0,1, \ldots, n, \\
\sum_{i=0}^{n} \alpha_{i}(t) & =1
\end{aligned}
$$

A portfolio is self-financing if the change in its value occurs only due to price changes, and is described by

$$
d y(t)=\sum_{i=0}^{n} v_{i}(t) d S_{i}(t)
$$


Substituting (1) and (2) in (6) and making use of the relations (4) and (5), one obtains:

$$
\begin{aligned}
& d y(t)=y(t)\left[\alpha_{0} r d t+\sum_{i=1}^{n} \alpha_{i}\left(\mu_{i} d t+\sum_{j=1}^{n} \sigma_{i j} d W_{j}\right)\right] \\
& =y(t)\left[r d t+\sum_{i=1}^{n} \alpha_{i}\left(\mu_{i}-r\right) d t+\sum_{j=1}^{n}\left(\sum_{i=1}^{n} \alpha_{i} \sigma_{i j}\right) d W_{j}\right]
\end{aligned}
$$

The portfolio value (7) is a controlled stochastic process with fractions of wealth $\alpha_{i}(t), i=1, \ldots, n$ as control variables. Assuming these to be constant over time, the optimal growth of $y(t)$ on the long run is achieved by maximizing its logmean at some instant of time $t$. Following the presentation in [8], we first derive the dynamics of $\ln [y(t)]$ from (7) using Ito's lemma as

$$
\begin{aligned}
d \ln y(t) & =\left[r+\sum_{i=1}^{n} \alpha_{i}\left(\mu_{i}-r\right)-\frac{1}{2} \sum_{j=1}^{n}\left(\sum_{i=1}^{n} \alpha_{i} \sigma_{i j}\right)^{2}\right] d t \\
& +\sum_{i=1}^{n} \alpha_{i} \sum_{j=1}^{n} \sigma_{i j} d W_{j}
\end{aligned}
$$

The log-optimal portfolio problem is that of selecting $\alpha_{i}$, $i=1,2, \ldots, n$, such that

$$
\max E\left[\ln \frac{y(t)}{y(0)}\right]
$$

is achieved for some $t$ (or equivalently, for every $t>0$ ), subject to (8). After integrating (8) and taking the expectation of the result, one obtains

$$
E\left[\ln \frac{y(t)}{y(0)}\right]=\left[r+\sum_{i=1}^{n} \alpha_{i}\left(\mu_{i}-r\right)-\frac{1}{2} \sum_{j=1}^{n}\left(\sum_{i=1}^{n} \alpha_{i} \sigma_{i j}\right)^{2}\right] t
$$

This shows that log-optimal fractions $\alpha_{i}, i=1,2, \ldots, n$, solve the following problem:

$$
\max \left[r+\sum_{i=1}^{n} \alpha_{i}\left(\mu_{i}-r\right)-\frac{1}{2} \sum_{j=1}^{n}\left(\sum_{i=1}^{n} \alpha_{i} \sigma_{i j}\right)^{2}\right]
$$

Forward difference with a sampling time $T$, gives the discrete form of (8) as

$$
\begin{aligned}
\Delta \ln y(k) & =\left[r+\sum_{i=1}^{n} \alpha_{i}\left(\mu_{i}-r\right)-\frac{1}{2} \sum_{j=1}^{n}\left(\sum_{i=1}^{n} \alpha_{i} \sigma_{i j}\right)^{2}\right] T \\
& +\sum_{i=1}^{n} \alpha_{i} \sum_{j=1}^{n} \sigma_{i j} \sqrt{T} e_{j}(k)
\end{aligned}
$$

where $e_{j}(k), j=1,2, \ldots, n$, are gaussian i.i.d. random variables with zero mean and variance one. It is clear from (12) that the values of $\alpha_{i}, i=1,2, \ldots, n$, such that

$$
\max E[\Delta \ln y(k)],
$$

or equivalently,

$$
\max E[\ln y(k+1)]
$$

is achieved for every $k$, are the log-optimal ones. This means that optimization problems (9), (13), and (14), all lead to solving (11).

\section{DYNAMICS OF ASSET HOLDINGS UNDER THE FINITE VARIATION CONSTRAINTS}

Let us introduce the no short-selling constraint as

Assumption(A1). Borrowing and short-selling of assets is not allowed: $\alpha_{i}(t), v_{i}(t) \geq 0, i=0,1, \ldots, n$.

The solution of (11) under the no short-selling constraints, will be denoted by $\alpha_{i}^{*}, i=0,1, \ldots, n$, and the corresponding logarithm of wealth as $\ln \left[y^{*}(k)\right]$. One can easily show that for such constant fractions of wealth, the trading strategies $v_{i}(t)$ have an infinite variation. This may contribute significantly to the transaction cost. Hence, in this section we will constrain the trading strategies to have a finite variation and be differentiable. This is a subset of a more general class of trading strategies of finite variation introduced in [3], [4].

Assumption(A2). Trading strategies have a finite logarithmic variation:

$$
d \ln \left[v_{i}(t)\right]=u_{i}(t) d t, i=0,1,2, \ldots, n,
$$

where $u(\cdot)$ is a continuous, adapted process.

In this case, the dynamics of fractions of wealth can be expressed as ${ }^{1}$

$$
d \ln \left[\alpha_{i}(t)\right]=u_{i}(t)+d \ln \left[S_{i}(t)\right]-d \ln [y(t)] .
$$

Using Ito's lemma, the dynamics of $\ln \left[S_{i}(t)\right]$ is obtained from (2) as

$$
d \ln \left[S_{i}(t)\right]=\mu_{i} d t-\frac{1}{2} \sum_{j=1}^{n} \sigma_{i j}^{2} d t+\sum_{j=1}^{n} \sigma_{i j} d W_{j}(t) .
$$

This means that (16) will always have noise terms, and the assumption of constant fractions of wealth can no longer be made. This also means there is no guarantee that the logoptimal performance $E\left[\ln y^{*}(k+1)\right]$ can be reached. Thus, the aim will be to minimize the following error

$$
e(k+1)=\ln y^{*}(k+1)-\ln y(k+1)
$$

where $\ln y(k+1)$ corresponds to a portfolio with finite varying trading strategies. An upper bound on $e(k+1)$ can be found using Jensen's inequality [10], as follows. Let $\gamma_{i}(k+1), i=0,1, \ldots, n$, be variables such that $0 \leq$ $\gamma_{i}(k+1) \leq 1$, and

$$
\sum_{i=0}^{n} \gamma_{i}(k+1)=1
$$

\footnotetext{
${ }^{1}$ See the proof of Lemma 2 for a similar derivation.
} 
Then the following holds for each $k$

$$
\ln [y(k+1)] \geq \sum_{i=0}^{n} \gamma_{i}(k+1) \ln \left[y_{i}(k+1)\right] .
$$

An upper bound on the error $e_{u}(k+1) \geq e(k+1)$ can thus be expressed as

$$
e_{u}(k+1)=\ln y^{*}(k+1)-\sum_{i=0}^{n} \gamma_{i}(k+1) \ln \left[y_{i}(k+1)\right]
$$

The objective is to make this upper bound as close to zero as possible. One way of achieving it is to constrain the variance to be zero and minimize the mean (other approaches are also possible). Due to $\log$-optimality of $\ln \left[y^{*}(k+1)\right]$ we have that

$$
E\left[\ln y^{*}(k+1)\right] \geq E\left[\sum_{i=0}^{n} \gamma_{i}(k+1) \ln y_{i}(k+1)\right],
$$

and thus $E\left[e_{u}(k+1)\right]$ is minimized if

$$
\max E\left[\sum_{i=0}^{n} \gamma_{i}(k+1) \ln y_{i}(k+1)\right]
$$

is achieved for each $k$. In order to find $\operatorname{Var}\left[e_{u}(k+1)\right]$, we need the dynamics of logarithmic asset holdings values $\ln \left[y_{i}(k+1)\right]$ under the finite variation constraints. These are derived in continuous time setting below.

Lemma 1: Portfolio is self-financing under assumptions (A1) and (A2) if

$$
\sum_{i=0}^{n} y_{i}(t) u_{i}(t) d t=0
$$

Proof : Applying Ito's lemma to (3) under assumption (A2) one obtains

$$
d y(t)=\sum_{i=0}^{n} v_{i}(t) d S_{i}(t)+\sum_{i=0}^{n} S_{i}(t) d v_{i}(t)
$$

Substituting the self-financing equation (6), we have

$$
\sum_{i=0}^{n} S_{i} d v_{i}=\sum_{i=0}^{n} S_{i}(t) v_{i}(t) \frac{d v_{i}(t)}{v_{i}(t)}=\sum_{i=0}^{n} y_{i}(t) d \ln \left[v_{i}(t)\right]=0
$$

Lemma 2: Let $x_{i}(t)=\ln \left[y_{i}(t)\right], i=0,1,2 \ldots, n$. Then, in a self-financing portfolio, under the assumptions (A1) and (A2), the following holds

$$
\begin{aligned}
& d x_{0}(t)=-\sum_{i=1}^{n} e^{x_{i}(t)-x_{0}(t)} u_{i}(t) d t+r d t \\
& d x_{i}(t)=u_{i}(t) d t+\mu_{i} d t-\frac{1}{2} \sum_{j=1}^{n} \sigma_{i j}^{2} d t+\sum_{j=1}^{n} \sigma_{i j} d W_{j}(t)
\end{aligned}
$$

Proof : First consider the case when $i=1,2, \ldots, n$. Taking the logarithm of (4), which is allowed due to assumption (A1), we obtain

$$
\ln \left[y_{i}(t)\right]=\ln \left[v_{i}(t)\right]+\ln \left[S_{i}(t)\right]
$$

The differential of (27) is

$$
d \ln \left[y_{i}(t)\right]=d \ln \left[v_{i}(t)\right]+d \ln \left[S_{i}(t)\right]
$$

which after substituting (15) and (17) gives equations (26). Similarly, we obtain the dynamics of $\ln \left[y_{0}(t)\right]$ as

$$
\begin{aligned}
d \ln \left[y_{0}(t)\right] & =d \ln \left[v_{0}(t)\right]+d \ln \left[S_{0}(t)\right] \\
& =-\sum_{i=1}^{n} e^{\ln \left[y_{i}(t)\right]-\ln \left[y_{0}(t)\right]} u_{i}(t) d t+r d t,
\end{aligned}
$$

where we have used the self-financing condition (22) in the form

$$
d \ln \left[v_{0}(t)\right]=-\sum_{i=1}^{n} e^{\ln \left[y_{i}(t)\right]-\ln \left[y_{0}(t)\right]} u_{i}(t) d t .
$$

Remark 1: Note that there are no explicit constraints on the state variables $x_{i}(t), i=0,1, \ldots, n$, or on the control variables $u_{i}(t), i=1,2, \ldots, n$, of equations (25) and(26).

Using the forward difference approximation with a sampling time $T$, we obtain the discrete form of (25) and (26) as

$$
\begin{aligned}
& \Delta x_{0}(k)=-\sum_{i=1}^{n} e^{x_{i}(k)-x_{0}(k)} u_{i}(k) T+r T \\
& \Delta x_{i}(k)=u_{i}(k) T+\mu_{i} T-\frac{1}{2} \sum_{j=1}^{n} \sigma_{i j}^{2} T+\sum_{j=1}^{n} \sigma_{i j} \sqrt{ } T e_{j}(k)
\end{aligned}
$$

Theorem 1: For $k=0,1, \ldots$, the variance of $e_{u}(k+1)$ is zero, iff we select

$$
\gamma_{i}(k+1)=\alpha_{i}^{*}
$$

for each $i=0,1, \ldots, n$, where $\alpha_{i}^{*}$ solve (11) under the no short-selling constraints.

Proof : Substituting the log-optimal logarithm of wealth $\ln \left[y^{*}(k+1)\right]$ from (12) in (19), together with $x_{0}(k+1)$ and $x_{i}(k+1), i=1,2, \ldots, n$, from (32) and (33), respectively, we obtain

$$
\operatorname{Var}\left[e_{u}(k+1)\right]=\sum_{j=1}^{n}\left[\sum_{i=1}^{n}\left(\alpha_{i}^{*}-\gamma_{i}(k+1)\right) \sigma_{i j}\right]^{2} T .
$$

It is clear that a sufficient condition for (35) to be equal to zero, is for (34) to hold. The necessary conditions for (35) to be zero are

$$
\sum_{i=1}^{n}\left(\alpha_{i}^{*}-\gamma_{i}(k+1)\right) \sigma_{i j}=0
$$

for every $j=1,2, \ldots, n$. This system of equations can also be written as

$$
\sigma^{\prime} D=0
$$

where $D=\left[\alpha_{1}^{*}-\gamma_{1}(k+1), \ldots, \alpha_{n}^{*}-\gamma_{n}(k+1)\right]^{\prime}$. Since the volatility matrix $\sigma$ is nonsingular, the system of equations (36) has a unique solution given by $D=0$, which gives (34) for each $k$. 
Due to (20), the error bound $e_{u}(k+1)$ with $\gamma_{i}(k+1)=\alpha_{i}^{*}$, is not zero in general, although its variance is. Combining (34) and (21) we obtain the criterion than minimizes $e_{u}(k+1)$ as

$\max E\left[\sum_{i=0}^{n} \gamma_{i}^{*} \ln y_{i}(k+1)\right]=\max E\left[\sum_{i=0}^{n} \gamma_{i}^{*} x_{i}(k+1)\right]$

for each $k=0,1, \ldots$, where ${ }^{2} \gamma_{i}^{*}=\alpha_{i}^{*}$.

\section{MAIN RESULT}

In order to give the investor the means for trade off between lower transaction cost and higher profit, we extend the criterion (37) to include a penalty on the logarithmic rates of change of trading strategies $u_{i}(k)$, and the resulting optimization problem can be stated as follows.

Portfolio control problem. Let $b_{i}(k), i=1,2, \ldots, n$, be some known positive variables, and the objective function be defined as

$$
V(k)=E\left[\sum_{i=0}^{n} \gamma_{i}^{*} \Delta x_{i}(k)-\sum_{i=1}^{n} b_{i}(k) u_{i}^{2}(k) T\right],
$$

where the states $\Delta x_{i}(k)$ are given by (32) and (33). The control problem is that of finding $u_{i}(k), i=1,2, \ldots$, that maximizes $V(k)$, for every $k=0,1,2, \ldots$.

For simplicity, we use $\Delta x_{i}(k)$ in (38) rather than $x_{i}(k+1)$ as in (37), which results in identical controls $u_{i}^{*}(k)$. Also, without loss of generality, we will deal with constant penalty coefficients $b_{i}(k)=b_{i}$, where $b_{i}$ is some known positive constant. Since the criterion (38) minimizes an upper bound on the error between the log-optimal portfolio and the one with finite variation constraints, we propose to call the resulting portfolio pseudo-log-optimal. The controls are now the logarithmic rates of change of trading strategies, rather than the fractions of wealth allocated across the assets.

Theorem 2: The solution to portfolio control problem always exists, is unique, and for every $k=0,1,2, \ldots$, is given by

$$
u_{i}^{*}(k)=\frac{1}{2 b_{i}}\left[\gamma_{i}^{*}-\gamma_{0}^{*} e^{x_{i}(k)-x_{0}(k)}\right], i=1,2, \ldots, n .
$$

Proof : The objective function (38) is

$$
\begin{array}{r}
V(k)=\left[-\sum_{i=1}^{n} e^{x_{i}(k)-x_{0}(k)} u_{i}(k) T+r T\right] \gamma_{0}^{*} \\
+\sum_{i=1}^{n} \gamma_{i}^{*}\left[u_{i}(k) T+\mu_{i} T-\frac{1}{2} \sum_{j=1}^{n} \sigma_{i j}^{2} T\right]-\sum_{i=1}^{n} b_{i} u_{i}^{2}(k) T
\end{array}
$$

Taking the partial derivatives with respect to $u_{i}(k)$, for every $i=1,2, \ldots, n$, and equating to zero, we obtain

$$
\frac{\partial V(k)}{\partial u_{i}(k)}=-e^{x_{i}(k)-x_{0}(k)} \gamma_{0}^{*} T+\gamma_{i}^{*} T-2 b_{i} u_{i}(k) T=0
$$

\footnotetext{
${ }^{2}$ We use this notation for clarity.
}

These equations always have a unique solution given by (39). The elements of the Hessian $H$ of $V(k)$ are given by:

$$
[H]_{i j}=\left\{\begin{aligned}
0 & : \quad i \neq j \\
-2 b_{i} T & : \quad i=j
\end{aligned}\right.
$$

Since $b_{i}>0$, the Hessian is negative definite. Thus, (39) is the maximum of $V(k)$.

Remark 2: Optimal controls (39) contain the fractions of wealth $\gamma_{i}^{*}=\alpha_{i}^{*}, i=0,1,2, \ldots, n$, from the log-optimal portfolio. This means that one needs to solve (11) under the no short-selling constraints before implementing (39).

The optimal trading strategies $v_{i}^{*}(k+1), i=0,1, \ldots, n$, for $k=0,1, \ldots, n$, are found by applying the forward difference to (15) and (31), and using optimal controls $u_{i}^{*}(k)$ from (39) to obtain

$$
\begin{aligned}
& v_{i}^{*}(k+1)=v_{i}^{*}(k) e^{u_{i}^{*}(k) T}, i=1,2, \ldots, n . \\
& v_{0}^{*}(k+1)=v_{0}^{*}(k) e^{u_{0}^{*}(k) T},
\end{aligned}
$$

where

$$
u_{0}^{*}(k)=-\sum_{i=1}^{n} e^{x_{i}(k)-x_{0}(k)} u_{i}^{*}(k)
$$

Equations (43) and (44), do not give an answer on how to make the initial optimal selection $v_{i}^{*}(0), i=0,1, \ldots, n$, which is understandable since the optimization has been carried out with respect to logarithmic rates of change rather than the quantities themselves. Thus, we make the initial selection identical with the log-optimal portfolio, which gives $e(0)=\ln y^{*}(0)-\ln y(0)=0$. In this case we have

$$
v_{i}^{*}(0)=\frac{\alpha_{i}^{*} y(0)}{S_{i}(0)}, i=0,1, \ldots, n .
$$

Remark 3: The controls in (39) will have the same form for any value of $T$. In particular, as $T$ approaches zero, the control in (39) approaches a continuous function (with $k$ replaced by $t$ ). The optimal trading strategies are then derived by solving equations in (15) with initial conditions given by (45).

\section{BOUNDS ON TRADING STRATEGIES AND THE PROBLEM OF RESTRICTING THE NUMBER OF SHARES}

From (39) is clear that the logarithmic variations are finite. The following result gives bounds on those variations.

Lemma 3: Lower and upper bounds on the optimal logarithmic changes $\Delta \ln v_{i}^{*}(k)=\ln \left[v_{i}^{*}(k+1)\right]-\ln \left[v_{i}^{*}(k)\right]$, $i=0,1,2, \ldots, n$, are

$$
\begin{aligned}
\frac{-T}{2} \frac{\max \left(\gamma_{i}^{*}\right) y(k)}{\min \left(b_{i}\right) y_{0}(k)} & \leq \Delta \ln v_{0}^{*}(k) \leq \frac{T \gamma_{0}^{*}}{2 \min \left(b_{i}\right)} \frac{y^{2}(k)}{y_{0}^{2}(k)} \\
\frac{-T \gamma_{0}^{*}}{2 b_{1}} \frac{y_{i}(k)}{y_{0}(k)} & \leq \Delta \ln v_{i}^{*}(k) \leq \frac{T \gamma_{i}^{*}}{2 b_{i}}
\end{aligned}
$$

where $\max \left(\gamma_{i}^{*}\right)$ and $\min \left(b_{i}\right)$ represent the maximal $\gamma_{i}^{*}$ and minimum $b_{i}$ for $i=1,2, \ldots, n$, respectively.

Proof : First we prove (46). The lower bound is found by starting from the discrete form of (24) and making use of 
(39) as

$$
\begin{aligned}
0 & =\sum_{i=0}^{n} y_{i}(k) \Delta \ln v_{i}^{*}(k) \leq \sum_{i=1}^{n} y_{i}(k) \frac{T \gamma_{i}^{*}}{2 b_{i}}+y_{0}(k) \Delta \ln v_{0}^{*}(k) \\
& \leq \frac{T \max \left(\gamma_{i}^{*}\right)}{2 \min \left(b_{i}\right)} \sum_{i=1}^{n} y_{i}(k)+y_{0}(k) \Delta \ln v_{0}^{*}(k) \\
& =\frac{T \max \left(\gamma_{i}^{*}\right)}{2 \min \left(b_{i}\right)}\left[y(k)-y_{0}(k)\right]+y_{0}(k) \Delta \ln v_{0}^{*}(k) . \\
\Delta & \ln v_{0}^{*}(k) \geq \frac{T \max \left(\gamma_{i}^{*}\right)}{2 \min \left(b_{i}\right)}\left[1-\frac{y(k)}{y_{0}(k)}\right] \geq \frac{-T \max \left(\gamma_{i}^{*}\right) y(k)}{2 \min \left(b_{i}\right) y_{0}(k)}
\end{aligned}
$$

Similarly, we find the upper bound as

$$
\begin{gathered}
0=\sum_{i=0}^{n} y_{i}(k) \Delta \ln v_{i}^{*}(k) \geq \sum_{i=1}^{n} \frac{-T \gamma_{0}^{*} y_{i}^{2}(k)}{2 b_{i} y_{0}(k)}+y_{0}(k) \Delta \ln v_{0}^{*}(k) \\
\geq-\frac{T \gamma_{0}^{*}}{2 \min \left(b_{i}\right) y_{0}(k)} \sum_{i=1}^{n} y_{i}^{2}(k)+y_{0}(k) \Delta \ln v_{0}^{*}(k) \\
\Delta \ln v_{0}^{*}(k) \leq \frac{T \gamma_{0}^{*}}{2 \min \left(b_{i}\right)} \frac{y^{2}(k)}{y_{0}^{2}(k)}
\end{gathered}
$$

Bounds in (47) follow directly from (43) and (39).

An important application of the upper bounds is when we restrict the number of shares per asset, where for some deterministic $M_{i}(k), k=1,2, \ldots$, it is required that

$$
v_{i}^{*}(k) \leq M_{i}(k)
$$

for every $i=0,1,2, \ldots, n$. Considering the sampling time $T$ as fixed, the penalty coefficients $b_{i}, i=1,2, \ldots, n$, can be selected as follows, in order for (48) to hold. First note that the upper bounds in (46) and (47) can be expressed as

$$
\begin{aligned}
& v_{0}^{*}(k+1) \leq v_{0}^{*}(k) \exp \left[\frac{T \gamma_{0}^{*} y^{2}(k)}{2 \min \left(b_{i}\right) y_{0}^{2}(k)}\right] \\
& v_{i}^{*}(k+1) \leq v_{i}^{*}(k) \exp \left(\frac{T \gamma_{i}^{*}}{2 b_{i}}\right), i=1,2, \ldots, n .
\end{aligned}
$$

Comparing these with (48), one can see that sufficient conditions for $b_{i}, i=1,2, \ldots, n$ (which in general can be time varying), to satisfy for every $k=1,2, \ldots$, are

$$
\begin{aligned}
\frac{T \gamma_{0}^{*} y^{2}(k)}{2 \min \left(b_{i}\right) y_{0}^{2}(k)} & \leq \ln \left[\frac{M_{0}(k+1)}{v_{0}^{*}(k)}\right] \\
\frac{T \gamma_{i}^{*}}{2 b_{i}} & \leq \ln \left[\frac{M_{i}(k+1)}{v_{i}^{*}(k)}\right]
\end{aligned}
$$

For the special case of unrestricted number of shares for the risk free asset (e.g. the bank account) and of constant restriction on the remaining assets $M_{i}(k)=M_{i}, i=$ $1,2, \ldots, n$, we have the following

Lemma 4: Let the initial selection be such that $v_{i}^{*}(0)<M_{i}$ for every $i=1,2, \ldots, n$. Then the upper constraints

$$
v_{i}^{*}(k) \leq M_{i}
$$

are satisfied for every $k=1,2, \ldots$, if

$$
b_{i} \geq \frac{T \gamma_{i}^{*}}{2 \ln \left[\frac{M_{i}}{v_{i}(0)}\right]}
$$

Proof : Referring to (50), for $k=1,2, \ldots$, we have

$$
v_{i}^{*}(k) \leq v_{i}^{*}(0) \exp \left(\frac{k T \gamma_{i}^{*}}{2 b_{i}}\right) .
$$

A sufficient condition for (53) is

$$
\begin{array}{r}
v_{i}^{*}(0) \exp \left(\frac{k T \gamma_{i}^{*}}{2 b_{i}}\right) \leq M_{i} \\
\exp \frac{T \gamma_{i}^{*}}{2 b_{i}} \leq\left[\frac{M_{i}}{v_{i}^{*}(0)}\right]^{\frac{1}{k}}
\end{array}
$$

Due to assumption $\left[M_{i} / v_{i}(0)\right]>1$, the above inequality yields

$$
\exp \frac{T \gamma_{i}^{*}}{2 b_{i}} \leq\left[\frac{M_{i}}{v_{i}^{*}(0)}\right]
$$

for every $k=1,2, \ldots$, and hence the result in (54).

One solution to the problem of having $v_{i}^{*}(0)<M_{i}$, $i=1,2, \ldots, n$, is to solve the log-optimal portfolio problem (11) under assumption (A1), with additional constraints

$$
\alpha_{i}<\frac{S_{i}(0) M_{i}}{y(0)}, i=1,2, \ldots, n .
$$

\section{Simulation EXAMPLE}

Let us consider a market having a bank account with $r=0.04, S_{0}(0)=1$, and a single stock with $\mu=0.05$, $\sigma=0.25$, and an initial price of $S_{1}(0)=1$. We choose the sampling time as $T=0.004$, and the initial capital $y(0)=1$. Log-optimal portfolio gives $\alpha_{1}^{*}=0.16$, and $v_{0}^{*}(0)=0.84, v_{1}^{*}(0)=0.16$. The control law (39) becomes

$$
u_{1}^{*}(k)=\frac{1}{b_{1}}\left[0.16-0.84 \frac{v_{1}(k) S_{1}(k)}{v_{0}(k) S_{0}(k)}\right] .
$$

Let us also have two different values of penalty coefficients, $b_{1}^{(1)}=0.05$ and $b_{1}^{(2)}=0.5$. In a market with no transaction cost, for one realization of the stock price, the trading of stock for the log-optimal and pseudo-log-optimal portfolios are shown in Fig.1. The trading takes place during the interval of time $[0,10]$. The total portfolio wealth is shown in Fig.2, where one can notice an almost undistinguishable behavior of portfolios at the beginning of trading period. In Fig.3, the end period portfolio wealth is enlarged. We assume there is no transaction cost for the bank account. The transaction costs that would have accumulated at time $(k+1) T$ for the log-optimal $C_{l}(k+1)$ and pseudo-logoptimal $C_{p}(k+1)$ portfolios, are assumed to be:

$$
\begin{aligned}
& C_{l}(k+1)=C_{l}(k)+0.01 \alpha^{*}\left|y^{*}(k+1)-y^{*}(k) S_{1}^{*}(k+1)\right| \\
& C_{p}(k+1)=C_{p}(k)+0.01\left|v_{1}^{*}(k+1)-v_{1}^{*}(k)\right| S_{1}(k)
\end{aligned}
$$




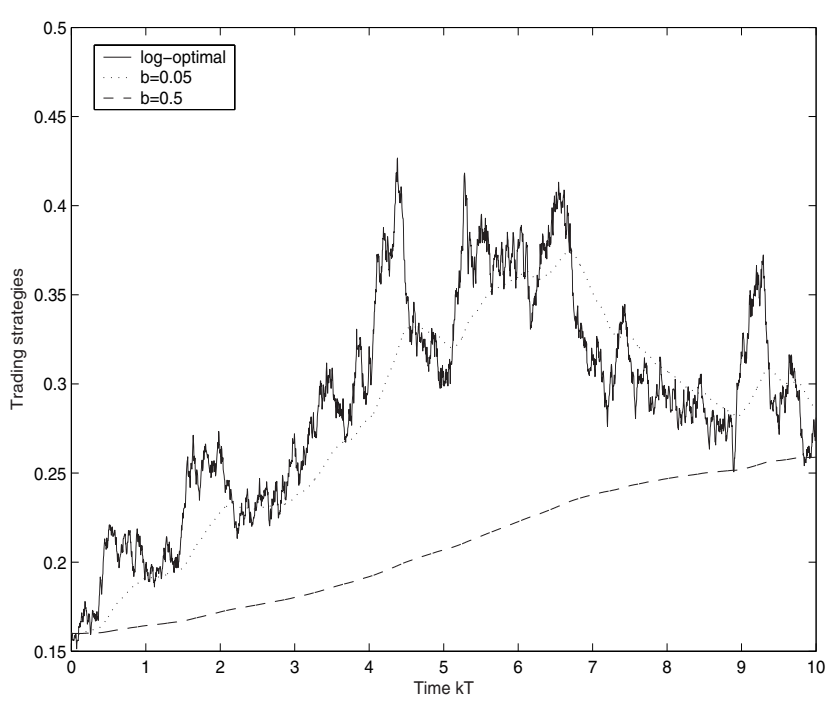

Fig. 1. Trading strategies (number of shares) of stock for logoptimal and pseudo-log-optimal portfolios.

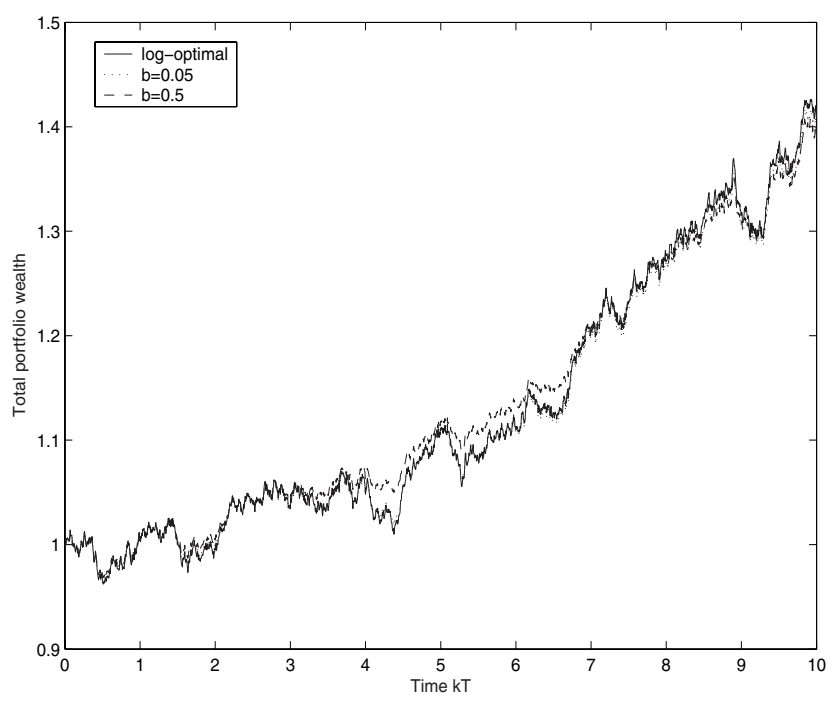

Fig. 2. Total portfolio wealth during the trading period.

with $C_{l}(0)=C_{p}(0)=0.01 v_{1}^{*}(0) S_{1}(0)$, and $S_{1}^{*}(k+1)=S_{1}(k+$ $1) / S_{1}(k)$. This corresponds to a charge of $1 \%$ of the total transaction value of buying or selling. The total wealth $y_{f}$ and transaction cost $C_{f}$ at the end of trading period are:

$$
\begin{aligned}
\mathbf{L o g}-\text { optimal: } y_{f} & =1.40838, C_{f}=0.04953 \\
\mathbf{b}_{\mathbf{1}}^{(\mathbf{1})}: y_{f} & =1.39561, C_{f}=0.00407 \\
\mathbf{b}_{\mathbf{1}}^{(\mathbf{2})}: y_{f} & =1.38984, C_{f}=0.00218
\end{aligned}
$$

This shows that for almost the same final wealth, the transaction cost is more than 12 and 22 times smaller in pseudo-log-optimal portfolios in comparison with the log-optimal one.

\section{CONCLUSIONS}

A new method for portfolio control with no short selling has been suggested which constrains the trading strategies

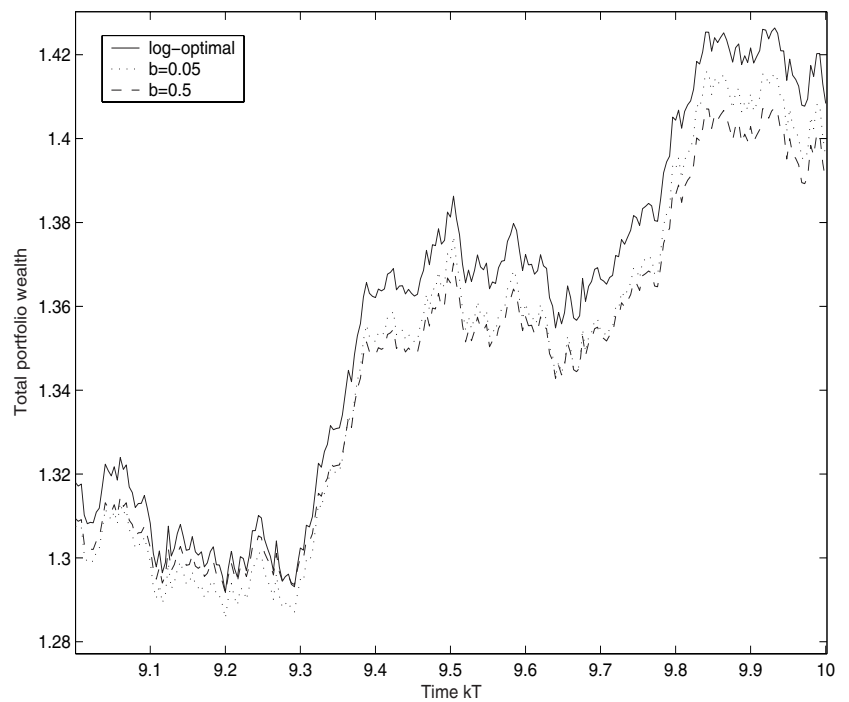

Fig. 3. Total portfolio wealth at the end of trading period.

to have a finite variation. This method minimizes an upper bound on the error between the log-optimal portfolio and the one with finite variation constraints. A penalty on the logarithmic rates of change is also included, offering the investor the means for trade off between lower transaction cost and higher profit. Explicit formulae are found for the optimal number of shares to be held. Bounds on the finite varying trading strategies are suggested and used for proposing a solution to the problem of restricting the number of shares per asset. For one realization of the stock price, a simulation example illustrates a significant reduction in transaction cost in comparison with the log-optimal portfolio, while maintaining almost the same total wealth. Extension of these results to log-mean-variance ([8], [9]) and power utility portfolios ([6], [8]), as well as explicit inclusion of transaction cost in the model, are the focus of current research.

\section{REFERENCES}

[1] L. Breiman, Optimal gambling systems for favorable games, Fourth Berkeley Symposium, vol. I, 65-78, 1961.

[2] G. N. Iyengar and T. M. Cover, Growth optimal policies with transaction costs, Proceedings of the IEEE International Symposium on Information Theory, August 1998.

[3] Y. M. Kabanov, Hedging and liquidation under transaction costs in currency markets, Finance and Stochastics, 3, pp. 237-248, 1999.

[4] Y. M. Kabanov and G. Last, Hedging under transaction cost in currency markets: a continuous-time model, Mathematical Finance, Vol. 12, No. 1, pp. 63-70, January, 2002.

[5] J. L. Kelly, Jr., A new interpretation of information rate, Bell System Technical Journal, 35, pp. 917-926, 1956.

[6] R. Korn, Optimal portfolios : stochastic models for optimal investment and risk management in continuous time, World Sientific, 1997.

[7] H. Latane, Criteria for choice among risky ventures, Journal of Political Economy, 67, pp. 144-155, 1959.

[8] D. G. Luenberger, Investment Science, Oxford University Press, 1998.

[9] D. G. Luenberger, A preference foundation for log mean-variance criteria in portfolio choice problems. Journal of Economic Dynamics and Control, 17, pp. 887-906, 1993.

[10] A. W. Roberts and D. E. Varberg, Convex functions, Academic Press, 1973. 